Fukushima J. Med. Sci., Vol. 59, No. 1, 2013

\title{
[Review]
}

\section{RECENT PROGRESS AND NEW PERSPECTIVES IN LYMPHOMA GLYCOBIOLOGY}

\author{
OSAMU SUZUKI and MASAFUMI ABE \\ Department of Diagnostic Pathology, School of Medicine, Fukushima Medical University, Fukushima, \\ Japan
}

\begin{abstract}
Glycosylation has recently become one of the most significant subjects in tumor biology, and cell surface glycosylation is closely associated with various biological phenomena in tumor cells. However, the biological significance of cell surface glycosylation and sialic acid linked to glycans in human malignant lymphoma is not well elucidated. We have determined that 1) sialylation or loss of $\mathrm{N}$-glycosylation is closely associated with a worse prognosis in human diffuse large B-cell lymphoma (DLBCL), and 2) glycosylation or sialic acid on the surface of lymphoma cells plays significant roles in cell adhesion or invasion to the extracellular matrix, cell growth, apoptosis and cell death. In the present review, the biological functions of glycosylation or sialic acid in human malignant lymphoma are discussed.
\end{abstract}

Key words : human malignant lymphoma, glycosylation, sialic acid.

\section{BACKGROUND}

Glycosylation and sialic acid on the surface of tumor cells affects cell-to-cell or cell-to-extracellular component interactions. Sialic acid is a sugar with increased expression in virus-transformed or chemically induced cancer cell lines, a phenomenon originally referred to as the Warren-Glick phenomenon $^{1)}$. Sialic acid plays important roles in the behaviors of tumor cells, including cell adhesion, invasiveness and metastasis ${ }^{2-5)}$, and it influences the patients' clinical outcomes ${ }^{6,7)}$. The role of sialic acid as a biological mask of cell surface structures is well documented ${ }^{8}$. The antirecognition effect of sialic acid can be explained by its negative charge, and oversialylation of malignant cells protects them from humoral or cellular defense systems and thus increases their malignancy ${ }^{9,10)}$. Prevention of malignant tumor cells from apoptosis or cell death by the masking effect of sialic acid may lead to more aggressive behaviors of tumor cells. Sialylation of cell surface glycoconjugates in malignant hematopoietic cells regulates their adhesive properties to extracellular matrix $(\mathrm{ECM})^{4}$. In addition, some lectin-reactive glycoconjugates have been associ- ated with the aggressiveness or metastatic capacity of malignant tumors. Sialylation of soybean agglutinin and Vicia villosa agglutinin lectin-reactive glycoconjugates on the cell surface is closely correlated with the highly metastatic phenotype of a murine or human lymphoma cell line $e^{3,5)}$. Additionally, cell surface sialylation regulates Fas-induced apoptosis in lymphoma cells ${ }^{11-13)}$.

Several sialyltransferases regulate cell surface sialylation $^{14)}$, and uridine diphosphate- $\mathrm{N}$-acetylglucosamine2-epimerase (UDP-GlcNAc2-epimerase), which catalyzes the rate-limiting step in sialic acid biosynthesis, is an important regulator of cell surface sialylation in human hematopoietic cell lines ${ }^{15,16}$.

Cell surface glycosylation includes $\mathrm{N}-$ and $\mathrm{O}-\mathrm{gl}$ ycosylation. $\mathrm{N}$-glycosylation is associated with cell $\operatorname{adhesion}^{17)}$, metastasis ${ }^{18)}$, carcinogenesis ${ }^{19,20)}$ and patient prognosis ${ }^{21)}$. Dennis JW et al. reported that the expression of L-PHA-reactive oligosaccharide, which is an $\mathrm{N}$-glycan that regulates cell adhesion to $\mathrm{ECM}$, is positively correlated to the distant metastasis of murine lymphoma cells ${ }^{22}$. Galectin is an endogenous lectin that reacts to cell surface glycans, such as the $\beta$-galactose residues of lactosamine structures $^{23,24)}$. The interaction between galectins

Corresponding author: Osamu Suzuki E-mail address : osuzuki@fmu.ac.jp

https://www.jstage.jst.go.jp/browse/fms http:/www.fmu.ac.jp/home/lib/F-igaku/ 
and cell surface glycans regulates cell growth or apoptosis of lymphocyte or lymphoma cells, and these regulatory functions are important in immune reactions ${ }^{25-30)}$. Galectins also regulate tumor cell adhesion $^{31)}$. Galectin-1 plays an important role in cell adhesion, and ligands for galectin-1 are lactosamine structures of cell surface oligosaccharides in ovarian cancer cells ${ }^{32}$. These data suggest that interactions between endogenous galectins and oligosaccharide ligands may be biologically significant in immune reaction and tumor biology.

\section{Heterogeneity of L-PHA-reactive oligosaccharide expression in human lymphoma}

Expression and sialylation of L-PHA-reactive oligosaccharide, an $\mathrm{N}$-glycan, are closely associated with clinical outcome in patients with $\mathrm{DLBCL}^{6,7)}$. DLBCL was grouped into three types: Group A, the non-reactive type in which L-PHA-reactive oligosaccharides are not expressed; Group B, the sialylated type in which the expression of sialylated L-PHA-reactive oligosaccharides is demonstrated by L-PHA lectin histochemical study ; and Group C, the non-sialylated type in which non-sialylated L-PHA-reactive oligosaccharides are expressed (Figure 1(a)). The survival of patients in Group A $+B$ or Group B was significantly shorter than that of patients in Group C (Figure 1b, 1c). Thus, the loss of non-sialylated L-PHA-reactive oligosaccharides or sialylation of L-PHA-reactive oligosaccharides is closely associated with a worse prognosis in DLBCL. N-acetylglucosaminyltransferase V (GnTV) catalyzes the synthesis of $\beta 1-6$ branching of L-PHA-reactive oligosaccharides. Expression of GnT-V is not always correlated with the expression of L-PHA-reactive oligosaccharides in DLBCL, and the loss of non-sialylated L-PHA-reactive oligosaccharides due to the lack of expression of GnT-V on lymphoma cells may be associated with aggressive DLBCL. Furthermore, sialylation of L-PHAreactive oligosaccharides is closely associated with a worse prognosis in DLBCL patients ; this association suggests that sialylation is related to the aggressiveness of DLBCL lymphoma cells. Recent reviews have reported that negatively charged sialic acid induces the oversialylation of malignant cells, which protects them from humoral or cellular defense systems ${ }^{10}$. Masking of the cell surface by sialic acid may lead to the escape of lymphoma cells from immuno-surveillance systems and may cause a worse prognosis for highly sialylated cases of DLBCL. Furthermore, $\alpha 2,6$-linked sialic acid residues were predominantly involved in sialylation of
L-PHA-reactive oligosaccharides of Group B. The $\alpha 2,6$-linked sialylated $\mathrm{N}$-linked oligosaccharides are involved in masking structures essential for the triggering of lymphocytes by B-cell lymphoma in the mixed lymphocyte reaction ${ }^{33)}$, and $\beta$-galactoside $\alpha 2,6$-sialyltransferase regulates the expression of cell surface differentiation antigens, such as HB-6, CDw75, CD76 and CD22, in B lymphocytes and B-cell malignancy ${ }^{34-36)} \cdot \quad \alpha 2,6$-sialylation of cell surfaces was responsible for reduced homotypic aggregation of colorectal carcinoma cells ${ }^{37}$, and $\mathrm{N}$-glycans bearing terminal $\alpha 2,6$-linked sialic acids enhanced the invasive potential of colon cancer cells ${ }^{38)} . \quad \alpha 2,6^{-}$ sialylation is regulated by $\alpha 2,6$-sialyltransferase, and its mechanism is crucial for B-cell maturation and antibody production in $\beta$-galactoside- $\alpha 2,6$-sialyltransferase (ST6Gal I) knockout mice ${ }^{39}$. Recently, we reported that $\alpha 2,6$-sialylation of cell surface $\mathrm{N}^{-}$ glycosylation inhibits galectin-1-mediated cell adhe$\operatorname{sion}^{40)}$, and cell surface sialylation inhibits galectin3 -induced apoptosis in the DLBCL cell line, HBL$2^{411}$. Based on these data, it is possible that the regulatory roles of sialylation of cell surface $\mathrm{N}$-glycans in galectin-mediated biological phenomena may be closely associated with malignant behaviors of human lymphoma cells.

Dennis et al. reported that overexpression of L-PHA-reactive oligosaccharides was associated with metastasis of a murine T-cell lymphoma line in animal models and that a lymphoma cell clone that had increased binding reactivity for L-PHA lectin on the cell surface showed a high metastatic rate $^{22}$. Candidate carrier proteins that have L-PHAreactive oligosaccharides are lysosomal-associated membrane proteins (LAMPs). Our preliminary data showed differences in the molecular weight of LAMP-1 among the DLBCL cases (data not shown), and it is possible that glycosylation of LAMP-1 may be different among the DLBCL cases. LAMPs expressed on the surface of murine T-cell lymphoma cells reduce the adhesion of the cells to ECM, leading to high metastatic capacity in vivo ${ }^{42}$. It is possible that differential glycosylation of LAMPs may be associated with altered cell adhesion to ECM among human DLBCL cases.

\section{Sialylation in cell adhesion to ECM and metastasis}

In the human Burkitt lymphoma cell line HBL8 , two clones were isolated by limiting dilutions of lymphoma cells. The 3G3 clone was highly sialylated, and the 3D2 clone was hyposialylated according to lectin-flow cytometric analysis ${ }^{5)}$. When HBL-8 cells were subcutaneously inoculated 

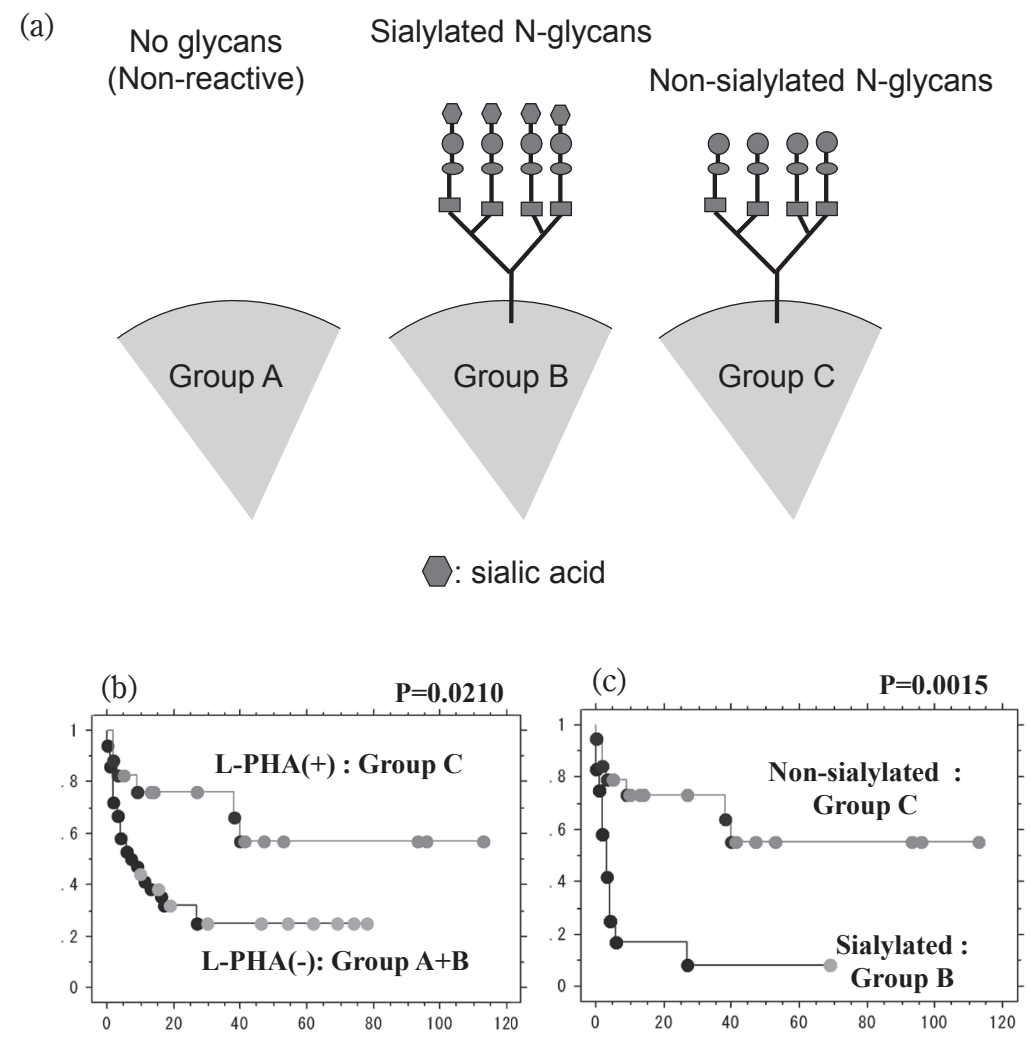

Fig. 1. (a) Schematic representation of heterogeneity of L-PHA reactive oligosaccharides in DLBCL. (b) and (c) DLBCL survival curves. Survival curves were established according to the Kaplan-Meier method and P-values were calculated based on Wilcoxon's test. The horizontal line represents months of survival, and the vertical line represents survival rate. The survival of patients in Group $\mathrm{A}+\mathrm{B}$ and Group B was significantly shorter than that of patients in Group C. (b) $P=0.0210$, Group A+B vs. Group C. (c) $P=0.0015$, Group B vs. Group C.

into severe combined immunodeficiency (SCID) mice, the $3 \mathrm{G} 3$ clone frequently metastasized to the spleen, ovary and bone marrow while the 3D2 clone had a low frequency of metastasis. Thus, cell surface sialylation of lymphoma cells is closely associated with high metastatic ability in the SCID mouse model. To determine why the highly sialylated clone has a high metastatic rate, we focused on the relationship between cell surface sialylation and the adhesive properties of $3 \mathrm{G} 3$ and $3 \mathrm{D} 2$ to $\mathrm{ECM}^{16)}$. From flow cytometric analyses the cell surface of the 3G3 clone was highly sialylated and that of the 3D2 clone was hyposialylated. Moreover, cell surface sialic acid content was significantly greater in the $3 \mathrm{G} 3$ clone than in the 3D2 clone. Cell surface sialylation in the $3 \mathrm{G} 3$ clone cells might reduce their attachment to collagen type IV and fibronectin as compared to the 3D2 clone in in vitro adhesion assays. Sialic acid metabolic complementation assays with several precursors of sialic acid revealed that hyposialylation in the 3D2 clone resulted from no mRNA expression of UDP-GlcNAc2-epimerase, which is a key enzyme in sialic acid biosynthesis
(Figure 2). From the RT-PCR analyses the expression of UDP-GlcNAc2-epimerase mRNA was correlated with sialic acid content among the two clones of HBL-8. Therefore, expression of UDPGlcNAc2-epimerase mRNA may regulate the expression of sialoglycoconjugates, which affect the adhesion of lymphoma cells to collagen type IV and fibronectin resulting in the different metastatic capacity in vivo.

In adhesion assays, the number of $3 \mathrm{G} 3$ clone cells attached to collagen type IV and fibronectin was less than that of the 3D2 clone, and neuraminidase treatment increased the number of 3G3 clone cells attached to collagen IV and fibronectin. Although both clones expressed some of the $\beta 1$-integrin family $(\alpha 1 \beta 1, \alpha 3 \beta 1, \alpha 4 \beta 1)$ on their cell surfaces (data not shown), there was a significant difference in their adhesive properties to collagen type IV and fibronectin. These data indicate that reduction in the attachment of $3 \mathrm{G} 3$ clone cells to fibronectin and collagen type IV resulted from cell surface sialylation and that the adhesive properties of these two clones to collagen type IV and fibronec- 


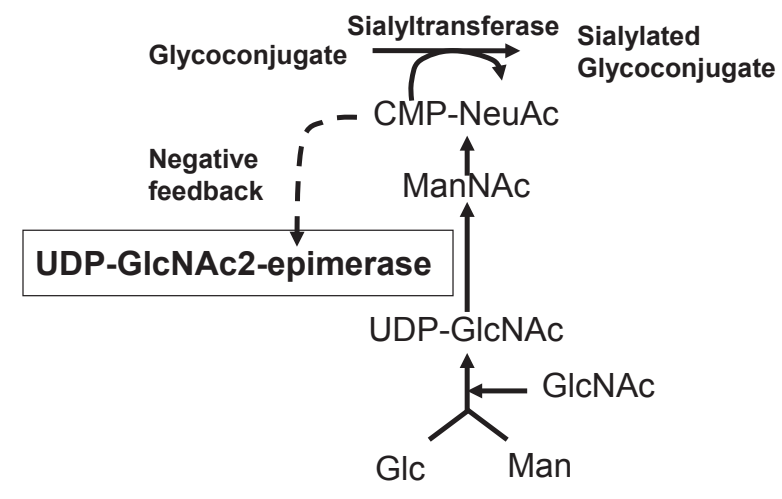

Fig. 2. Schematic representation of the sialic acid biosynthetic pathway. UDP-GlcNAc2-epimerase catalyzes the rate-limiting step in the biosynthesis of sialic acid. The activity of UDP-GlcNAc2epimerase is negatively regulated by CMP-NeuNAc. Abbreviations : NeuAc (N-acetyl neuraminic acid), CMP-NeuAc (cytidine monophosphate$\mathrm{N}$-acetyl neuraminic acid), ManNAc (N-acetyl-Dmannosamine), UDP-GlcNAc (uridine diphosphate$\mathrm{N}$-acetyl-D-glucosamine), GlcNAc (N-acetyl-Dglucosamine), Glc (D-glucose), Man (D-mannose).

tin is regulated by cell surface sialylation rather than the expression of $\beta 1$-integrins. We previously reported that sialylation of L-PHA-reactive oligosaccharides in human DLBCL was associated with a worse prognosis or a more advanced clinical stage of the disease ${ }^{6,7)}$. Cell adhesion to ECM is closely associated with the metastatic capacity of malignant tumor cells ${ }^{4}$. Therefore, alteration of the cell adhesive capacity by cell surface sialylation may explain the different metastatic capacity of lymphoma cells. Inhibition of cell adhesion to ECM by cell surface sialylation may lead to more release of lymphoma cells from the primary site in the metastatic pathway. It is possible that sialylation of L-PHA-reactive oligosaccharides in lymphoma cells may play an important role in the regulation of lymphoma cell attachment to ECM, leading to a high metastatic capacity and advanced clinical stage in DLBCL cases.

The previous studies showed that UDPGlcNAc2-epimerase played a crucial role in the regulation of sialic acid biosynthesis in human hematopoietic cell lines ${ }^{15,16)}$ and that UDP-GlcNAc2epimerase is a crucial factor in mouse development ${ }^{43}$. In a metabolic complementation assay, the hyposialylated 3D2 clone cultivated with $\mathrm{N}$-acetyl-Dmannosamine (ManNAc) had increased expression of sialoglycoconjugates, but this was not the case with GlcNAc or Man, which enter the sialic acid biosynthetic pathway upstream of ManNAc (Figure 2). These data suggest that hyposialylation in the
3D2 clone is due to a decrease in UDP-GlcNAc2epimerase activity. On the other hand, cultivation of the highly sialylated 3G3 clone with ManNAc, GlcNAc or Man did not alter lectin-binding activities. This result is probably due to the negative feedback mechanism in which cytidine monophosphate-N-acetyl neuraminic acid (CMP-NeuAc) inhibits the function of UDP-GlcNAc2-epimerase, which keeps the level of sialic acid biosynthesis constant (Figure 2). The levels of ST6Gal I and ST3Gal IV mRNAs were the same in both clones, but UDP-GlcNAc2-epimerase mRNA was detected in the highly sialylated 3G3 clone and not in the hyposialylated 3D2 clone. These data suggest that hyposialylation in the 3D2 clone is due to a loss of UDP-GlcNAc2-epimerase mRNA expression.

In the invasion assay, the $3 \mathrm{G} 3$ clone was more invasive in Matrigel than the 3D2 clone. Matrigel is composed of laminin, collagen type IV, entactin and heparan-sulfate proteoglycans ${ }^{44}$. The highly invasive properties of the $3 \mathrm{G} 3$ clone in Matrigel may be attributable to its reduction in adhesion to collagen type IV. Sialylation of the cell surface is closely correlated with the highly metastatic phenotype of human Burkitt's lymphoma cell line HBL-8 in animal models ${ }^{5}$. We speculate that cell surface sialylation may reduce the attachment of lymphoma cells to collagen type IV, leading to more invasion into the basement membrane and the capacity to invade Matrigel. This increased capability for invasion may facilitate the release of lymphoma cells from the primary site into the blood stream through the basement membrane in vivo.

In summary, we examined the significance of sialylation in lymphoma cell adhesion and invasion by using human Burkitt's lymphoma cell lines. The expression of sialoglycoconjugates and the content of cell surface sialic acid, which modulate the adhesive properties of Burkitt's lymphoma cells to ECM, appeared to be regulated by UDP-GlcNAc2epimerase at the transcriptional level.

\section{Sialylation in Fas-induced apoptosis}

Programmed cell death (apoptosis) has become a critical topic in tumor cell biology. Both Keppler and Peter et al. reported that differential sialylation of cell surface glycoconjugates in a human B-cell lymphoma cell line regulates susceptibility to Fasmediated apoptosis ${ }^{11,12)}$. These data suggest that sialic acid is a key regulator that induces apoptosis of tumor cells. But, the detailed mechanisms of intracytoplasmic signal transduction by desialylation in Fas-induced apoptosis are unclear. We demon- 
strated that apoptosis of Jurkat $\mathrm{T}$ cells induced by incubation with anti-Fas monoclonal antibody $\mathrm{CH} 11$ was enhanced by pre-treatment with neuraminidase, which cleaves cell surface sialic $\operatorname{acid}^{13)}$. The enhancement of Fas-induced apoptosis by pre-treatment with neuraminidase was inhibited by $\mathrm{z}-\mathrm{VAD}-$ fmk, a broad caspase inhibitor, and Ac-LEHD-CHO, an inhibitor of caspase- 9 , but not by Ac-IETD-CHO, an inhibitor of caspase- 8 and -6 , imipramine, an inhibitor of acidic sphingomyelinase, glutathione, an inhibitor of neutral sphingomyelinase, or Fumonisin B1, an inhibitor of ceramide synthase. The loss of mitochondrial membrane potentials $(\Delta \psi \mathrm{m})$ involved in Fas-induced apoptosis was enhanced by pretreatment with neuraminidase. Furthermore, from the western blot analysis, pre-treatment with neuraminidase resulted in a reduction of the molecular weight of Fas of about $8 \mathrm{kDa}$. These data suggest that the enhancement of Fas-induced apoptosis by pre-treatment with neuraminidase was mediated by a caspase-9-dependent pathway closely associated with the loss of $\Delta \psi \mathrm{m}$, not by activation of caspase- 8 or -6 , acidic and neutral sphingomyelinases, or ceramide synthase. It is possible that sialic acid linked to glycoconjugates of Fas may regulate Fasinduced apoptosis in human $\mathrm{T}$-cell lymphoma. Sialic acids on the cell surface, especially those linked to glycoconjugates of Fas, appeared to be inhibitory for Fas-induced apoptosis of Jurkat cells.

Fas is expressed on the cell surface as a trimer, and its monomer has two or three $\mathrm{N}$-glycosylation sites $^{45,46)}$, and those $\mathrm{N}$-glycans are sialylated ${ }^{11,12)}$. Western blot analysis indicated that pre-treatment with neuraminidase resulted in a reduction in the molecular weight of Fas of about $8 \mathrm{kDa}^{13)}$. Therefore, sialic acids of Fas are removed after neuraminidase treatment, and those alterations in the sialylation of Fas glycans resulted in the modulation of signal transduction mediated by Fas in Fasinduced apoptosis.

Fas-induced apoptosis is classified into two types. Type II apoptosis is mediated by the activation of caspase- 9 and the loss of $\Delta \psi \mathrm{m}^{47,48)}$ and is characterized by weak caspase- 8 activation at the death-inducing signaling complex. Our study demonstrated that the enhancement of Fas-induced apoptosis by removal of sialic acids from Fas is closely associated with the activation of caspase- 9 and loss of $\Delta \psi \mathrm{m}$, but not with the activation of caspase -8 or -6 . These data suggest that the caspase-9 and mitochondrial-dependent pathway is mainly involved in Fas-induced apoptosis in Jurkat

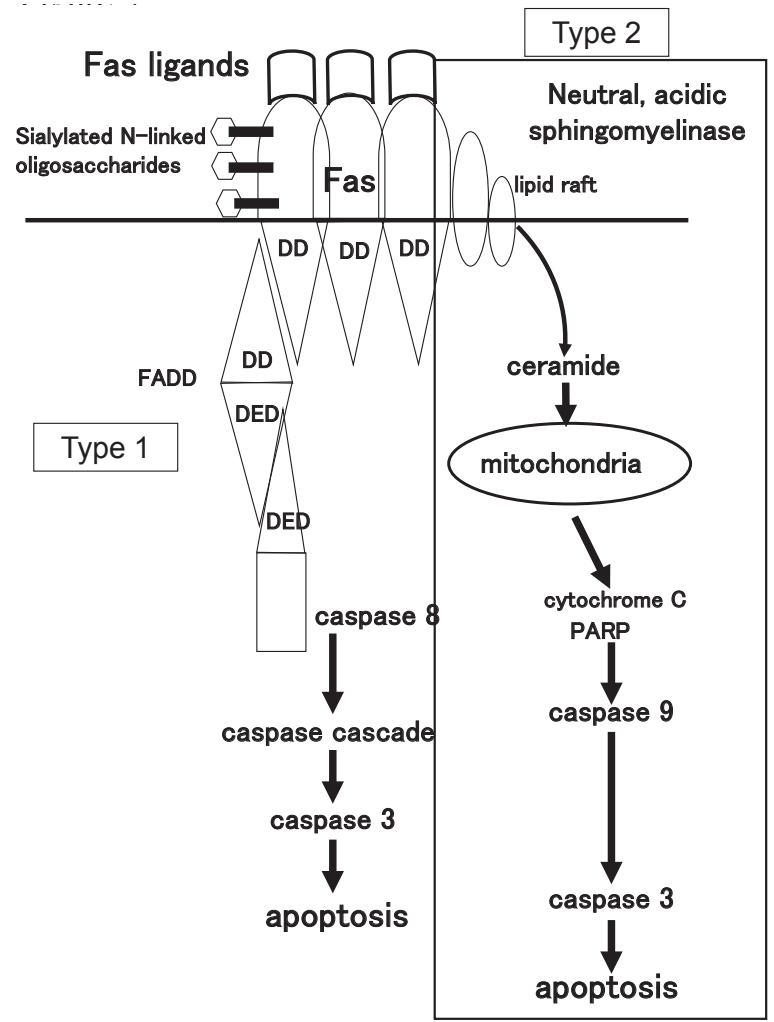

Fig. 3. Schematic representation of the regulation of Fas-induced apoptosis by sialylation in Jurkat cells. Loss of sialic acid from Fas molecule enhances Fas-induced apoptosis through type II apoptotic pathway. DD : death domain; DED : death effecter domain; FADD: Fas associated death domain.

cells and that the alteration in sialylation of Fas affects only the caspase- 9 and mitochondrial-dependent pathway (type II apoptosis) (Figure 3).

Ceramide is reported to play a role in apoptotic signals initiated through the Fas system in various types of cells, but the role of ceramide in Fasinduced apoptosis is controversial ${ }^{49-52}$. Our study showed no inhibitory effects of glutathione, imipramine and Fumonisin B1 on Fas-induced apoptosis of Jurkat cells. Thus, the data indicate that the production of ceramide by neutral or acidic sphingomyelinases or ceramide synthase is not involved in Fas-induced apoptosis of Jurkat cells and the enhancement of Fas-induced apoptosis by treatment with neuraminidase. In summary, Fas-induced apoptosis appeared to be regulated by sialic acid in the Fas molecule. Cell surface sialylation or sialic acid on Fas can protect lymphoma cells from Fasinduced apoptosis. Oversialylation of tumor cells can protect tumor cells from various stimuli ${ }^{10)}$, and it may result in protection of lymphoma cells from Fas-induced apoptosis. This mechanism may pro- 
vide a new insight that high levels of sialylation can inhibit Fas-induced apoptosis in human malignant lymphoma.

\section{Galectin-1-induced cell death}

CD45 is a heavily glycosylated protein having many L-PHA reactive oligosaccharides that react with L-PHA lectin, leading to the blastic transformation of $\mathrm{T}$ lymphocytes ${ }^{53)}$. Not only $\mathrm{N}$-glycans but also O-glycans on CD45 are ligands for galectin- $1^{54,55)}$. Galectin-1 induces apoptosis of T lymphocytes through the inactivation of CD45 protein tyrosine phosphatase (PTP), and alteration in glycosylation of CD45 affects galectin-1-mediated apoptosis and induces growth inhibition of Burkitt's lymphoma cell lines ${ }^{29)}$. In our preliminary experiments, galectin-1 induced reduction of CD45 PTP activity in a human anaplastic large cell lymphoma cell line, H-ALCL, which was established in our laboratory (unpublished data). This result suggested that galectin-1 may interact with CD45 and inhibit CD45 PTP activity, resulting in the induction of cell death ; thus, the galectin-1 and CD45 PTP system may be a mechanism for the induction of cell death in human lymphoma cells. To clarify the functions of $\mathrm{N}$ - and O-glycans in DLBCL, we analyzed the regulatory roles of $\mathrm{N}$ - and $\mathrm{O}$-glycans in galectin-1-induced cell death in a DLBCL cell line, HBL-2 ; the alteration of $\mathrm{N}-$ and $\mathrm{O}$-glycans on $\mathrm{CD} 45$ was analyzed by Western blot ${ }^{55)}$. Galectin-1 induced cell death of HBL-2 in vitro. Swainsonine (SW) is a potent inhibitor of $\alpha$-mannosidase II which catalyzes the synthesis of complex type N-linked oligosaccharides, and benzyl-GalNAc (BZGalNAc) is a potent inhibitor of O-glycosylation. Treatment with SW or BZGalNAc prevented cell death of HBL-2 cells by galectin-1. Western blot analysis revealed that SW treatment reduced the molecular weight by about 5 $\mathrm{kDa}$ of one isoform at $190 \mathrm{kDa}$ among three isoforms of CD45. BZGalNAc treatment reduced the molecular weight of another isoform by about 15 $\mathrm{kDa}$. These data suggest that the amount of CD45 $\mathrm{N}$-glycans or O-glycans was reduced by SW and BZGalNAc treatment, respectively, and that the reduction of $\mathrm{CD} 45 \mathrm{~N}$-glycans or $\mathrm{O}$-glycans may prevent the interaction between CD45 and galectin- 1 in DLBCL cells.

L-PHA-reactive oligosaccharides synthesized by $\mathrm{GnT}-\mathrm{V}$ regulate $\mathrm{T}$-cell activation via $\mathrm{T}$-cell receptor signaling in the immune response through the interaction between a galactose-specific lectin, galectin-3, and $\mathrm{N}$-glycans of the $\mathrm{T}$-cell receptor ${ }^{30)}$. In addition, inhibition of cell surface $\mathrm{N}$-glycosylation in $\alpha$-mannosidase II gene knockout mice is closely associated with autoimmune disease ${ }^{56)}$. We reported previously that the expression patterns of $\mathrm{N}$-glycans synthesized by GnT-V correlated with the clinical outcome of human DLBCL ${ }^{7)}$. These data suggest that $\mathrm{N}$-glycans are closely associated with regulation of $\mathrm{T}$ lymphocyte activation in the immune reaction and the behavior of DLBCL. We demonstrated that SW treatment prevented the death of HBL-2 cells by galectin-1 and reduced the molecular weight of CD45. These data suggest that galectin-1 induces cell death of HBL-2 cells through its interaction with cell surface N-glycans. Furthermore, galectin-1 reacts with the terminal lactosamine structures of $\mathrm{N}$-glycans or O-glycans of CD45, leading to cell death in hematological malignancy ${ }^{29)}$. Thus, $\mathrm{N}$-glycans on the surface of HBL-2 cells appear to be essential for galectin-1-induced cell death, and it is possible that galectin-1 induces cell death of HBL-2 cells through its interaction with CD45 Nglycans. In addition to $\mathrm{N}$-glycans, O-glycans also play a significant role in galectin-1-mediated cell death $^{25)}$. BZGalNAc is a potent inhibitor of the O-glycosylation pathway and can modulate the functions of $\mathrm{O}$-glycosylated mucin in colonic carcinoma cells $^{57,58)}$. BZGalNAc treatment reduced the molecular weight of CD45 and prevented the cell death of HBL-2 cells by galectin- 1 . These data suggest that CD45 contains O-glycans and that cell death of HBL-2 cells by galectin- 1 may be mediated by CD45 O-glycans.

We reported previously that DLBCL cases without non-sialylated L-PHA-reactive oligosaccharides had a worse prognosis than those with nonsialylated L-PHA-reactive oligosaccharides ${ }^{6,7)}$. It is speculated that the loss of L-PHA-reactive oligosaccharides may prevent galectin-1-mediated cell death of lymphoma cells, leading to more aggressive behavior of DLBCL as shown in Figure 4. This proposed mechanism may imply different biological behaviors of lymphoma cells between the cases with and without $\mathrm{N}$-glycans in DLBCL.

\section{Galectin-1-mediated cell adhesion}

The biological role of sialic acid in galectin1-mediated cell adhesion still remains unclear. We analyzed the regulatory roles of cell surface sialylation in cell adhesion to galectin- 1 in the DLBCL cell line HBL-2, and the Burkitt's lymphoma cell line HBL- $8^{40)}$. Vibrio cholerae neuraminidase treatment enhanced HBL-2 cell adhesion to galectin-1, suggesting that sialic acid inhibits HBL-2 cell adhesion to galectin-1. The data from employing two 


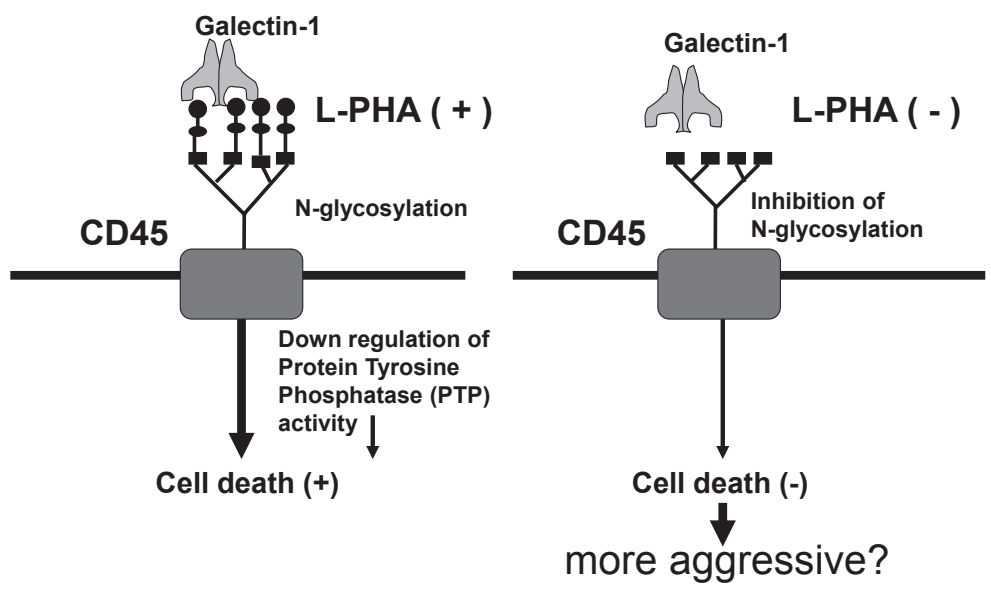

Fig. 4. Schematic representation of the regulatory roles of $\mathrm{N}$-glycosylation in the growth of lymphoma cells through the interaction between CD45 N-glycans and galectin-1. Loss of N-glycans in cell surface prevents galectin1 -induced cell death.

different neuraminidases, Vibrio cholerae and Newcastle disease virus neuraminidase, showed that $\alpha 2,6$-linked sialic acid plays an important role in the inhibition of HBL-2 cell adhesion to galectin1. In addition, neuraminidase treatment enhanced the cell adhesion to galectin- 1 much more with the highly sialylated HBL-8 $3 \mathrm{G} 3$ clone than that with the hyposialylated HBL-8 3D2 clone. Flow cytometric analysis revealed the expression of partially sialylated L-PHA-reactive oligosaccharides on the surfaces of HBL-2 cells. SW treatment decreases cell surface sialic acid in HBL-2 on flow cytometric analysis. SW treatment also enhanced HBL-2 cell adhesion to galectin-1. These data indicate that SW treatment decreased sialylated L-PHA-reactive oligosaccharides, resulting in cell surface desialylation and leading to enhancement of cell adhesion to galectin-1. Therefore, alteration of cell surface sialylation or $\mathrm{N}$-glycan expression regulates cell adhesion to galectin-1 in human malignant lymphoma.

Among the various galectins, galectin- 1 acts as an ECM protein ${ }^{31)}$. For instance, human ovarian cancer cells can adhere to galaptin (synonym to galectin-1) through interactions with cell surface lactosamine structures, and cell surface sialylation inhibits the interaction between cell surface lactosamine and galaptin ${ }^{32}$. We demonstrated that cell surface sialylation of HBL-2 cells inhibited their adhesion to galectin-1 and that galectin-1 induced the death of HBL-2 cells. Therefore, in human B-cell lymphoma, sialylation of cell surface oligosaccharides appears to inhibit cell adhesion to galectin-1 and protect lymphoma cells from galectin-1-induced cell death. The number of adherent cells to galectin-1 increased dramatically with Vibrio cholerae neuraminidase treatment but not with Newcastle disease virus neuraminidase treatment. These data suggest that $\alpha 2,6$-linked sialic acid on the cell surface prevents HBL-2 cells from adhesion to galectin-1. $\alpha 2,6$-linked sialic acid inhibits galectin-1 binding to $\mathrm{T}$ cells ${ }^{27)}$, and galectin-1 binds $\alpha 2,3^{-}$ sialylated and non-sialylated terminal $\mathrm{N}$-acetyllactosamine units, but not $\alpha 2,6$-sialylated one ${ }^{59}$. These data suggest that the type of sialylation linkage affects the binding ability of galectin-1 to terminal residues of oligosaccharides. Furthermore, we reported previously that $\alpha 2,6$-linked sialylation of terminal residues of L-PHA-reactive oligosaccharides was closely associated with a worse prognosis for patients with $\mathrm{DLBCL}^{7}$. Taken together, it appears that the modulation of cell adhesion to galectin- 1 by cell surface $\alpha 2,6$-linked sialylation may affect the malignant behavior of human DLBCL. There is a possibility that modulation in cell adhesion to galectin- 1 by $\alpha 2,6$-linked sialylation of cell surface glycans may be associated with altered malignant behaviors, such as metastasis or invasion. In the other aspects cell surface sialylation can inhibit cell death induced by galectin-1 on cell adhesion to galectin-1 leading to cell survival or more aggressive behaviors of the highly sialylated cases in DLBCL. In our preliminary data, neuraminidase treatment or SW treatment dramatically enhanced cell adhesion to galectin-1 in the H-ALCL cell line (unpublished data). In conclusion, the regulatory roles of galectin-1-induced cell adhesion by cell surface sialylation may explain why the cases with sialylated $\mathrm{N}$-glycans showed a more advanced clinical stage than the cases with non-sialylated 
$\mathrm{N}$-glycans in $\mathrm{DLBCL}^{6)}$.

\section{Galectin-3-induced cell death}

Galectin-3 is a novel anti-apoptotic molecule containing a functional bcl-2 family BH1 (NWGR) domain $^{60)}$ that protects cells from apoptosis by inhibiting cytochrome $c$ release from mitochondria ${ }^{61)}$. Galectin-3 is extensively expressed in DLBCL, but not in other B cell lymphoma cell lines ${ }^{62}$. Intracytoplasmic galectin-3 expression can inhibit Fasinduced apoptosis ${ }^{63}$. When added to the exterior of cells, galectin-3 induces apoptosis by interacting with terminal galactose residues on cell surface glycans $^{63)}$. We showed that exogenous galectin-3 induces the death of HBL-2 human DLBCL cells ${ }^{411}$. Annexin $\mathrm{V}$ assays and morphological evaluation revealed that extracellular galectin-3 causes cell death by inducing apoptosis. Galectin- 3 interacts with LAMPs or with the glycans of CD45 $5^{64,65)}$.

We showed that SW treatment inhibits galectin3 -induced apoptosis in HBL-2 cells ${ }^{41}$. The data suggest that $\mathrm{N}$-linked oligosaccharides, such as L-PHA-reactive oligosaccharides, are responsible for the induction of lymphoma cell apoptosis by galectin-3. Therefore, loss of N-linked oligosaccharides may inhibit galectin-3-induced apoptosis, leading to more aggressive behavior in DLBCL. In addition, neuraminidase treatment enhanced galectin-3-induced apoptosis in HBL-2 cells. These results suggest that cell surface sialylation can protect lymphoma cells from galectin-3-induced apoptosis. We revealed that galectin-3 was expressed in macrophages in tissue sections of human lymphomas by performing a immunohistochemical study (data not shown). Therefore, galectin-3 may be produced by macrophages, and soluble galectin- 3 may react to cell surface glycans on lymphoma cells in a paracrine manner. In our reports, sialylation of L-PHA-reactive oligosaccharides has been closely associated with a worse prognosis of patients with $\mathrm{DLBCL}^{6,7)}$. Taken together, these findings suggest that protection of lymphoma cells from galectin3 -induced apoptosis by cell surface sialylation results in more aggressive behavior, at least in part, by enhancing tumor cell survival.

Galectin-3-mediated cell adhesion is closely associated with lung metastasis of melanoma cells in an experimental animal model, and interaction between poly $\mathrm{N}$-acetyllactosamine structures and galectin-3 is needed for melanoma cell metastasis ${ }^{66)}$. These data suggest that galectin-3 can play a significant role in cell adhesion as well as in induction of apoptosis, and galectin-3-mediated cell adhesion may alter the distant metastatic capacity of tumor cells. In future investigations, the biological roles of galectin-3 as ECM in human lymphoma cell adhesion must be clarified.

In conclusion, our results show that cell surface $\mathrm{N}$-glycans and sialylation play important roles in galectin-3-induced apoptosis. The regulatory mechanisms mediating galectin-3-induced apoptosis by cell surface $\mathrm{N}$-glycans and sialylation may provide a new scientific foundation in tumor cell biology of human malignant lymphoma and may imply that differences in the cell surface glycosylation pattern are associated with different biological behaviors among human malignant lymphoma cases.

\section{Sialylation and cell death}

Stress signals induce ceramide (Cer) through sphingomyelinase (SMase) activation, and metabolites of Cer such as sphingosine (Sph) and sphingosine-1-phosphate (S-1-P) play a significant role in many biological processes. Our previous study aims to elucidate the association between alteration in cell surface sialylation and Cer-induced cell death in the human Burkitt lymphoma cell line, HBL8. The highly sialylated $3 \mathrm{G} 3$ clone was less sensitive to $\mathrm{C} 6$-Cer-induced cell death. On the other hand, the hyposialylated 3D2 clone was more sensitive to C6-Cer-induced cell death. Neuraminidase treatment or knockdown by siRNA of UDPGlcNAc2-epimerase enhanced the amount of cell death induced by $\mathrm{C} 6-\mathrm{Cer}$ in the highly sialylated 3G3 clone. Sialic acid metabolic complementation assays using several precursors of sialic acid showed that cell surface resialylation by ManNAc inhibited C6-Cer-induced cell death in the hyposialylated 3D2 clone. In addition, clone 3G3 was less sensitive to Sph than the 3D2 clone. In conclusion, in human malignant lymphoma, Cer and its metabolitesinduced cell death is regulated by the amount of sialic acid on the cell surface, which in turn is regulated by mRNA expression of UDP-GlcNAc2epimerase $^{67)}$.

Goni et al. recently reported that Cer and sphingolipids modified the physical properties of the cell membrane ${ }^{68)}$, and Montes et al. reported that Cer induced an increased efflux or permeability in cell membranes ${ }^{69}$. LDH release assay revealed that Cer can induce the membrane permeability and neuraminidase pre-treatment enhanced LDH release in HBL-8 $3 \mathrm{G} 3$ clone. Therefore, the evidence suggests that Cer induces the enhancement of membrane permeability death and cell membrane permeability induced by Cer is regulated by cell sur- 
face sialylation. Neuraminidase treatment enhanced cell death induced by $\mathrm{C} 6$-Cer in the highly sialylated 3G3 clone. Furthermore, the sialic acid complementation assay using ManNAc in the hyposialylated 3D2 clone showed that cell surface resialylation by ManNAc complementation inhibited cell death induced by Cer. These data suggest that cell surface sialic acid can protect lymphoma cells from Cer-induced cell death. A highly sialylated $3 \mathrm{G} 3$ clone was more metastatic than a hyposialylated 3D2 clone in the metastatic SCID mouse model of human lymphoma cell lines ${ }^{5)}$. Lymphoma cells may be exposed to a stressful environment in the blood stream or in distant target organs upon metastasis, and various stresses induce Cer or sphingolipid, which is produced by the sphingomyelin pathway in vivo. In addition, the inhibition of cell death is reported to be a crucial characteristic of metastatic cancer cells ${ }^{70}$. Therefore, increased cell surface sialylation may reduce susceptibility of the $3 \mathrm{G} 3$ clone cells to Cerinduced cell death through the masking effects of lymphoma cells by sialic acids, leading to increased cell survival (Figure 5). Protection of lymphoma cells from stress-induced cell death by sialic acid masking may lead to the aggressive behaviors of lymphoma cells, such as metastasis. mRNA expression of UDP-GlcNAc2-epimerase correlates with the sialic acid content among two clones of HBL-8, $3 \mathrm{G} 3$ and $3 \mathrm{D} 2^{16)}$, and in our experiments, the knockdown by siRNA for UDP-GlcNAc2-epimerase for the highly sialylated $3 \mathrm{G} 3$ clone decreased cell surface sialylation and also enhanced C6-Cer-induced cell death. These data suggest that cell surface sialylation by expression of UDP-GlcNAc2-epimerase is closely associated with the inhibition of Cerinduced cell death and that lymphoma cells may escape from stress-induced cell death through cell surface sialylation regulated by UDP-GlcNAc2epimerase, leading to an increase of metastatic colonization of distant organs. Metastasis-related clonal selection by stress-mediated cell death may be decided by cell-surface sialylation.

In conclusion, cell death induced by Cer and its metabolites in a human Burkitt lymphoma cell line appears to be influenced by cell surface sialylation. Lymphoma cells can escape from many stressful conditions in vivo through cell surface masking and protection of lymphoma cells by sialylation, which is regulated by UDP-GlcNAc2-epimerase. Cell survival from stressful conditions by cell surface sialylation may be closely associated with prolonged cell survival of lymphoma cells leading to metastatic colonization at distant target organs. Those survival mechanisms due to protection of lymphoma cells by oversialylation explain the more malignant behaviors, such as high frequency in metastasis. This mechanistic model may provide a new scientific foundation in tumor cell biology of human malignant lymphoma.

Galectins in Hodgkin and anaplastic large cell lymphoma

Hodgkin lymphoma is classified into two categories, classical Hodgkin lymphoma (cHL) and nodular lymphocyte predominant Hodgkin lymphoma $(\text { NLPHL })^{71}$. Galectin-1 is a useful marker for differentiation between $\mathrm{cHL}^{-}$and NLPHL ${ }^{72}$. Galectin1 is induced by AP1-dependent transcriptional mechanisms. Galectin- 1 is expressed by $\mathrm{R}-\mathrm{S}$ cells and not expressed in DLBCL ${ }^{73)}$. Galectin-3 protects cells from apoptosis through interactions with bcl-2 by blocking alterations in mitochondrial mem-

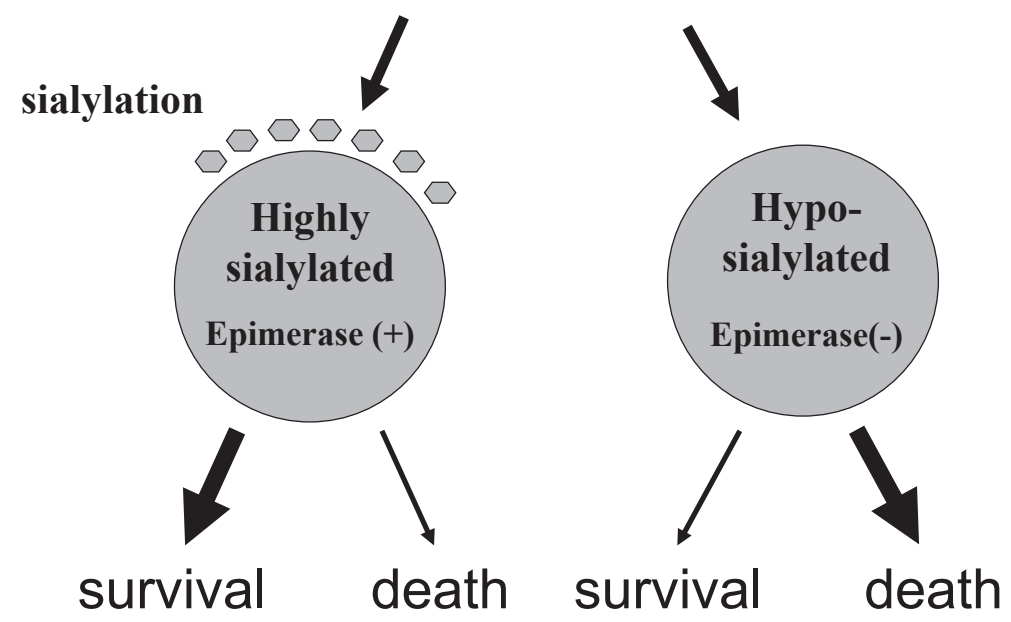

Fig. 5. A schematic representation of the regulatory mechanism of cell surface sialylation in ceramide-induced cell death. 
brane potentials. Galectin-3 is expressed in DLBCL, but not in Burkitt lymphoma ${ }^{74)}$. Galectin3 is a useful marker for differentiation between anaplastic large cell lymphoma (ALCL) and $\mathrm{HL}^{75)}$. The biological functions of galectins, which are released by Hodgkin lymphoma cells, still remain unclear. Galectins are produced by macrophages or R-S cells, and there is a possibility that those galectins may induce apoptosis of $\mathrm{R}-\mathrm{S}$ cells. Our recent tissue microarray analysis showed that all of the HL cases are galectin- 3 negative and almost all the ALCL cases are galectin-3 positive ${ }^{76)}$. Galectin-1 can induce apoptosis of HL cell lines and ALCL cell lines heterogeneously. The knockdown of galectin-3 by siRNA resulted in no alteration in galectin1-induced apoptosis of Karpas 299 cells suggesting that galectin-3 does not contribute to galectin-1-induced apoptosis of lymphoma cells in ALCL. On the other hand, when we transfected galectin-3 cDNA plasmid into human embryonic kidney 293 (HEK293) cells, marked aggregation of the HEK293 cells occurred $^{76)}$. The data suggested that galectin-3 plays an important role in cell-to-cell interactions. Furthermore, the epithelial membrane protein (EMA), which is one of the galectin-3 ligands, was expressed in many ALCL cell lines including the Karpas 299 cell line (in our preliminary data) and in some cases of ALCL ${ }^{77)}$. Taken together with these data there is a possibility that galectin-3 may act as a cell adhesion molecule in the interactions between galectin-3 and EMA. In future studies, we need to analyze the function of galectin- 3 in relation to cell-to-cell interactions in human lymphoma cells.

Furthermore, CD30 stimulation by CD30L or Ki-1 monoclonal antibody (mAb) dramatically enhances galectin-1-induced apoptosis in Karpas 299 cells. There is a possibility that an alternative signaling pathway by CD30 stimulation may be involved in galectin-1 induced apoptosis, although both anti-apoptotic roles and induction of cell death by CD30 stimulation were reported previously ${ }^{78)}$. In ALCL there is cross talk between galectin-1-induced apoptosis and CD30 stimulation, and these biological phenomena may be associated with tumor cell behaviors in ALCL. The recent clinical trials using $\mathrm{mAb}$ for $\mathrm{CD} 30$ showed that stimulation of CD30 can induce anti-tumor effects in $\mathrm{ALCL}^{79}$. The CD30 stimulation in combination with galectin-1 treatment may be more effective to lymphoma cells in ALCL.

\section{PERSPECTIVES}

There are many variations in biological functions of glycosylation in human malignant lymphoma. From our studies, many biological functions of glycosylation (metastasis in animal models, cell adhesion, invasion, cell growth, apoptosis and cell death) have been documented in human lymphoma. However, the biological functions of glycosylation have not yet been fully elucidated in Hodgkin lymphoma, B-cell lymphomas other than DLBCL, T-cell lymphoma or NK-cell lymphoma. Many aspects of glycosylation in these human lymphoma histological types still remain to be elucidated. In future investigations, we will clarify the biological functions of glycosylation in these histological subtypes.

In vitro biological assays revealed that the biological phenomena in lymphoma cell biology appeared to be strictly regulated by cell-surface glycosylation or sialylation. Cell surface glycans and sialic acids are not merely tumor markers ; rather, they are significant functional molecules in tumor cell biological phenomena. The masking effects of sialic acid on the cell surface or protection of lymphoma cells by cell surface sialylation is closely associated with malignant behaviors of lymphoma cells, and loss of $\mathrm{N}$-glycosylation resulted in the protection of lymphoma cells from galectin-glycanmediated cell death and is closely associated with aggressiveness of lymphoma cells, which is correlated to the patients' clinical outcome. Taken together, our broad research data shows that sialylation and glycosylation appear to have many universal roles in tumor cell biology of human malignant lymphoma. We hope that the current studies in this field provide new scientific foundations in the tumor biology of human malignant lymphoma and may be useful in the development of new therapeutic strategies.

\section{REFERENCES}

1. Warren L, Fuhrer JP, Buck CA. Surface glycoproteins of normal and transformed cells : a difference determined by sialic acid and a growth-dependent sialyl transferase. Proc Natl Acad Sci USA, 69(7) : 1838-1842, 1972.

2. Takano R, Muchmore E, Dennis JW: Sialylation and malignant potential in tumor cell glycosylation mutants. Glycobiology, 4 : 665-674, 1994.

3. Altevogt P, Fogel M, Cheingsong-Popov R, Dennis J, Robinson P, Schirrmacher V. Different patterns 
of lectin binding and cell surface sialylation detected on related high- and low-metastatic tumor lines. Cancer Res, 43 : 5138-5144, 1983.

4. Dennis JW, Waller C, Timpl R and Schirrmacher V. Surafce sialic acid reduces attachment of metastatic tumor cells to collagen type IV and fibronectin. Nature, 300 : 274-276, 1982.

5. Abe M, Suzuki O, Tasaki K, Tominaga K, Wakasa H. Analysis of lectin binding properties on human Burkitt's lymphoma cell lines that show high spontaneous metastasis to distant organs in SCID mice : the binding sites for soybean agglutinin lectin masked by sialylation are closely associated with metastatic lymphoma cells. Pathol Int, 46 : 977-983, 1996.

6. Suzuki O, Nozawa Y, Kawaguchi T, Abe M. Phaseolus vulgaris leukoagglutinating lectin-binding reactivity in human diffuse large B cell lymphoma and its relevance to the patient's clinical outcome: lectin histochemistry and lectin blot analysis. Pathol Int, 49 : 874-880, 1999.

7. Suzuki O, Nozawa Y, Kawaguchi T, Abe M. Alpha2,6-sialylation of L-PHA reactive oligosaccharides and expression of $\mathrm{N}$-acetyl glucosaminyltransferase $\mathrm{V}$ in human diffuse large $\mathrm{B}$ cell lymphoma. Oncol Rep, 10 : 1759-1764, 2003.

8. Klem S, Schauer R : Sialic acids in molecular and cellular interactions. Int Rev Cytol, 175 : 137-240, 1997.

9. Schauer R. The diversity of sialic acids and their interplay with lectins. Glycobiology, 135-149, 2007.

10. Schauer R. Sialic acids: fascinating sugars in higher animals and man. Zoology, 107 : 49-64, 2004.

11. Keppler OT, Peter ME, Hinderlich S, Moldenhauer G, Stehling P, Schmitz I, Schwartz-Albiez R, Reutter W, Pawlita M. Differential sialylation of cell surface glycoconjugates in a human B lymphoma cell line regulates susceptibility for CD95 (APO-1/Fas)-mediated apoptosis and for infection by a lymphotropic virus. Glycobiology, 9 : 557569, 1999.

12. Peter ME, Hellbardt S, Schwartz-Albiez R, Westendorp MO, Walczak H, Moldenhauer G, Grell M, Krammer PH. Cell surface sialylation plays a role in modulating sensitivity towards APO-1mediated apoptotic cell death. Cell Death Differ, 2 : 163-171, 1995.

13. Suzuki O, Nozawa Y, Abe M. Sialic acids linked to glycoconjugates of Fas regulate the caspase-9-dependent and mitochondria-mediated pathway of Fas-induced apoptosis in Jurkat $\mathrm{T}$ cell lymphoma. Int J Oncol, 23 : 769-774, 2003.

14. Dall'Olio F, Chiricolo M, Ceccarelli C, Minni F, Marrano D, Santini D. Beta-galactoside alpha2,6 sialyl transferase in human colon cancer : contri- bution of multiple transcripts to regulation of enzyme activity and reactivity with Sambucus nigra agglutinin. Int J Cancer, 88(1) : 58-65, 2000.

15. Keppler OT, Hinderlich S, Langner J, SchwartzAlbiez R, Reutter W and Pawlita M. UDP-GlcNAc 2-epimerase : a regulator of cell surface sialylation. Science, 284 : 1372-1376, 1999.

16. Suzuki O, Nozawa Y, Kawaguchi T, Abe M. UDPGlcNAc2-epimerase regulates cell surface sialylation and cell adhesion to extracellular matrix in Burkitt's lymphoma. Int J Oncol, 20 : 1005-1011, 2002.

17. Zheng M, Fang H, Hakomori S. Functional role of $\mathrm{N}$-glycosylation in $\alpha 5 \beta 1$ integrin receptor. J Biol Chem, 269 : 12325-12331, 1994.

18. Shao DM, Wang $\mathrm{QH}$, Chen $\mathrm{C}$, Shen $\mathrm{ZH}$, Yao M, Zhou XD, Tang ZY, Gu JX. N-acetylglucosaminylt ransferase $\mathrm{V}$ activity in metastatic models of human hepatocellular carcinoma in nude mice. J Exp Clin Cancer Res, 18 : 331-335, 1999.

19. Guo Hua-Bei, Zhang QS, Chen H. Effects of Hras and $\mathrm{v}$-sis overexpression on $\mathrm{N}$-acetylglucosaminyltransferase $\mathrm{V}$ and metastasis-related phenotypes in human hepatocarcinoma cells. J Cancer Res Clin Oncol, 126 : 263-270, 2000.

20. Miyoshi E, Nishikawa A, Ihara Y, Gu J, Sugiyama T, Hayashi N, Fusamoto H, Kamada T, Taniguchi N. $\mathrm{N}$-acetylglucosaminyltransferase III and $\mathrm{V}$ messenger RNA levels in LEC rats during hepatocarcinogenesis. Cancer Res, 53 : 3899-3902, 1993.

21. Akita H, Miyoshi E, Suzuki O, Itoh T, Katoh H, Taniguchi N Expression of N-acetylglucosaminyltransferase $\mathrm{V}$ is associated with prognosis and histology in Non-small cell lung cancers. Clin Cancer Res, 10 : 1773-1779, 2004.

22. Dennis JW, Laferté S, Waghorne C, Breitman ML, Kerbel RS. Beta 1-6 branching of Asn-linked oligosaccharides is directly associated with metastasis. Science, 236(4801) : 582-585, 1987.

23. Rabinovich GA, Rubinstein N, Toscano MA. Role of galectins in inflammatory and immunomodulatory processes. Biochimi Biophysi Acta, 1572 : 274-284, 2002.

24. Barondes SH, Castronovo V, Cooper DN, Cummings RD, Drickamer K, Feizi T, Gitt MA, Hirabayashi J, Hughes C, Kasai K, Leffler H, Liu FT, Lotan R, Mercurio AM, Monsigny M, Pillai S, Poirer F, Raz A, Rigby PWJ, Rini JM, Wang J. Galectins : a family of animal beta-galactosidebinding lectins. Cell, 76(4) : 597-598, 1994.

25. Perillo NL, Pace KE, Seilhamer JJ, Baum LG. Apoptosis of $\mathrm{T}$ cells mediated by galectin-1. Nature, 378 : 736-739, 1995.

26. Nguyen JT, Evans DP, Galvan M, Pace KE, Leitenberg D, Bui TN, Baum LG. CD45 modulates galectin-1-induced $\mathrm{T}$ cell death : regulation 
by expression of core 2 O-glycans. J Immunol, 167 : 5697-5707, 2001.

27. Amano M, Galvan M, He J, Baum LG. The ST6Gal I sialyltransferase selectively modifies $\mathrm{N}$-glycans on CD45 to negatively regulate galectin-1-induced CD45 clustering, phosphatase modulation, and T cell death. J Biol Chem, 278 : 74697475, 2003.

28. Demetriou M, Granovsky M, Quaggin S, Dennis JW. Negative regulation of T-cell activation and autoimmunity by Mgat5 N-glycosylation. Nature, 409 : 733-739, 2001.

29. Foullit M, Joubert-Caron R, Poirier F, Bourin P, Monostori E, Levi-Strauss M, Raphael M, Bladier D, Caron M. Regulation of CD45-induced signaling by galectin-1 in Burkitt lymphoma B cells. Glycobiology, 10 ; 413-419, 2000.

30. Demetriou M, Granovsky M, Quaggin S, Dennis JW. Negative regulation of T-cell activation and autoimmunity by Mgat $5 \mathrm{~N}$-glycosylation. Nature, 409 : 733-739, 2001.

31. He j, Baum LG. Presentation of galectin- 1 by extracellular matrix triggers $\mathrm{T}$ cell death. J Biol Chem, 279 : 4705-4712, 2004.

32. Skrincosky DM, Allen HJ, Bernacki RJ. Galaptinmediated adhesion of human ovarian carcinoma A121 cells and detection of cellular galaptin-binding glycoproteins. Cancer Res, 53 : 2667-2675, 1993.

33. Leland DP, Sideny WW, Gerald WH. Cell surface sialic acid influences tumor cell recognition in the mixed lymphocyte reaction. J Immunol, 139 : 262270, 1987.

34. Bast JEGB, Zhou LJ, Freeman GJ, Colley KJ, Ernst TJ, Munro JM, Tedder TF. The HB-6,CDw75, and CD76 differentiation antigens are unique cellsurface carbohydrate determinants generated by the $\beta$-galactoside $\alpha 2,6$-sialyltransferase. J Cell biol, 116 : 423-435, 1992.

35. Gross HJ, Merling A, Moldenhauer G, SchwartzAlbiez R. Ecto-sialyltransferase of human B lymphocytes reconstitutes differentiation markers in the presence of exogenous CMP-N-acetyl neuraminic acid. Blood, 87 : 5113-5126, 1996.

36. Sherry AW, Ruea YH, Jonathan E, XueLian L, Joseph TYL. Murine B cell differntiation is accompanied by programmed expression of multiple novel $\beta$-galactoside $\alpha 2,6$-sialyltransferase mRNA forms. Glycobiology, 10 : 67-75, 2000.

37. Bosch J, Brossmer R, Kemmner W, Schlag P. Preparation and characterization of differently aggregated colorectal carcinoma cell subpopulations from surgical specimens. Cancer Detect Prev, 22(4) : 319-329, 1998.

38. Zhu Y, Srivatana U, Ullah A, Gagneja H, Berenson CS, Lance P. Suppression of a sialylatransferase by antisense DNA reduces invasiveness of human colon cancer cells in vitro. Biochem Biophys Acta, 1536 : 148-160, 2001.

39. Hennet T, Chui D, Paulson JC, Marth JD. Immune regulation by the ST6Gal sialyltransferase. Proc Natl Acad Sci USA, 95(8): 4504-4509, 1998.

40. Suzuki O, Nozawa Y, Abe M. The regulatory roles of cell surface sialylation and $\mathrm{N}$-glycans in human B cell lymphoma cell adhesion to galectin-1. Int J Oncol, 28(1) : 155-160, 2006.

41. Suzuki O, Abe M. Cell surface N-glycosylation and sialylation regulates galectin-3-induced apoptosis in human diffuse large B cell lymphoma. Oncol Rep, 19: 743-748, 2008.

42. Laferte S, Dennis JW. Glycosylation-dependent collagen binding activities of two membrane glycoproteins in MDAY-D2 tumor cells. Cancer Res, 48 : 4743-4748, 1988.

43. Martina Schwarzkopf, Klaus-Peter Knobeloch,, Elvira Rohde, Stephan Hinderlich, Nicola Wiechens, Lothar Lucka, Ivan Horak, Werner Reutter, and Rüdiger Horstkorte. Sialylation is essential for early development in mice. Proc Natl Acad Sci USA, 99(8) : 5267-5270, 2002.

44. Kleinmen HK, McGarvey ML, Liotta LA, Robey PG, Tryggvason K. Isolation and characterization of type IV procollagen, laminin, and heparan sulfate proteoglycan from the EHS sarcoma. Biochemistry, 21 : 6188-6193, 1982.

45. Bajorath J. Analysis of Fas-ligand interactions using a molecular model of the receptor-ligand interface. J Computer-Aided Molecular Design, 13 : 409-418, 1999

46. Itoh N, Yonehara S, Ishii A, Yonehara M, Mizushima S, Sameshima M, Hase A, Seto Y, Nagata S. The polypeptide encoded by the cDNA for human cell surface antigen Fas can mediate apoptosis. Cell, 66 : 233-243, 1991.

47. Scaffidi C, Fulda S, Srinvasan A, Friesen C, Li F, Tomaselli KJ, Debatin KM, Krammer PH, Peter ME. Two CD95 (APO-1/Fas) signaling pathways. EMBO J, 17 : 1675-1687, 1998.

48. Cuvillier O, Edsall L, Spiegel S. Involvment of sphingosine in mitochondria-dependent Fas-induced apoptosis of type II Jurkat T cells. J Biol Chem, 275 : 15691-15700, 2000.

49. Sillence DJ, Allan D. Evidence against an early signaling role for ceramide in Fas-mediated apoptosis. Biochem J, 324 : 29-32, 1997.

50. Gamen S, Marzo I, Anel A, Pineiro A, Naval J. CPP32 inhibition prevents Fas-induced ceramide generation and apoptosis in human cells. FEBS Lett, 390 : 233-237, 1996.

51. Hsu S, Wu C, Luh T, Han S, Lai M. Apoptotoc signal of Fas is not mediated by ceramide. Blood, 91 : 2658-2663, 1998. 
52. Watts JD, Gu M, Polverino AJ, Patterson SD, Abersold R. Fas-induced apoptosis of $\mathrm{T}$ cells occurs independently of ceramide generation. Proc Natl Acad Sci USA, 94 : 7292-7296, 1998.

53. Monostori E, Hartyani Z, Ocsovszky I, Hegedus Z, Oravecz T, Kalman M, Ando I. Effect of phytohemagglutinin on CD45 in T cells. Immunol Lett, 42 : 197-201, 1994.

54. Pace KE, Lee C, Stewart PL, Baum LG. Restricted receptor segregation into membrane microdomains occurs on human T cells during apoptosis induced by galectin-1. J Immunol, 163 : 38013811, 1999.

55. Suzuki O, Nozawa Y, Abe M. Regulatory roles of altered $\mathrm{N}$ - and $\mathrm{O}$-glycosylation of $\mathrm{CD} 45$ in galectin-1-induced cell death in human diffuse large B cell lymphoma. Int J Oncol, 26 : 1063-1068, 2005.

56. Chui D, Sellakumar G, Green R, Sutton-Smith M, McQuistan T, Marek K, Morris H, Dell A, Marth J. Genetic remodeling of protein glycosylation in vivo induces autoimmune disease. P Natl Acad Sci USA, 98 : 1142-1147, 2001.

57. Gouyer V, Leteurtre E, Delmotte P, Steelant WFA, Krzewinski-Recchi M, Zanetta J, Lesuffleur T, Trugnan G, Delannoy P, Huet G. Differential effect of GalNAc $\alpha-\mathrm{O}$-bn on intracellular trafficking in enterocytic HT-29 and Caco-2 cells : correlation with the glycosyltransferase expression pattern. J Cell Sci, 114 : 1455-1471, 2001.

58. Hennebicq-reig S, Lesuffleur T, Capon C, Bolos C, Kim I, Moreau O, Richet C, Hemon B, Recchi M, Maes E, Aubert J, Real F, Zweibaum A, Delannoy P, Degand P, Huet G. Permanent exposure of mucin-secreting HT-29 cells to benzyl-N-acetyl$\mathrm{a}-\mathrm{D}$-galactosaminide induces abnormal $\mathrm{O}$-glycosylation of mucins and inhibits constitutive and stimulated MUC5AC secretion. Biochem J, 334 : 283-295, 1998.

59. Leppanen A, Stowell S, Blixt O, Cummings RD. Dimeric galectin-1 binds with high affinity to $\alpha 2,3-$ sialylated and non-sialylated terminal N-acetyllactosamine units on surface-bound extended glycans. J Biol Chem, 280 : 5549-5562, 2005.

61. Akahani S, Nangia-Makker P, Inohara H, Kim HR, Raz A. Galectin-3 : a novel antiapoptotic molecule with a functional BH1 (NWGR) domain of Bcl-2 family. Cancer Res, 57(23) : 5272-5276, 1997.

61. Yu F, Finery Jr RL, Raz A, Kim HRC. Galectin-3 translocates to the perinuclear membranes and inhibits cytochrome c release from the mitochondria. J Biol Chem, 277 : 15819-15827, 2002.

62. Hoyer KK, Pang M, Gui D, Shintaku IP, Kuwabara I, Liu FT, Said JW, Baum LG, Teitell MA. An antiapoptotic role for galectin-3 in diffuse large B-cell lymphomas. Am J Pathol, 164(3) : 893-902, 2004.

63. Fukumori T, Takenaka Y, Yoshii T, Kim HRC,
Hogan V, Inohara H, Kagawa S, Raz A. CD29 and CD7 mediate galectin-3-induced type II T-cell apoptosis. Cancer Res, 63 : 8302-8311, 2003.

64. Sarafian V, Jadot M, Foidart JM, Letesson JJ, Van den Brule F, Castronovo V, Wattiaux R, Coninck SW. Expression of Lamp-1 and Lamp-2 and their interactions with galectin-3 in human tumor cells. Int J Cancer, 75(1) : 105-111, 1998.

65. Stillman BN, Hsu DK, Pang M, Brewer CF, Johnson P, Liu FT, Baum LG. Galectin-3 and galectin-1 bind distinct cell surface glycoprotein receptors to induce $\mathrm{T}$ cell death. J Immunol, 76(2) : 778-789, 2006.

66. Srinivasan N, Bane SM, Ahire SD, Ingle AD, Kalraiya RD. Poly N-acetyllactosamine substitutions on $\mathrm{N}$ - and not $\mathrm{O}$-oligosaccharides or Thomsen-Friedenreich antigen facilitate lung specific metastasis of melanoma cells via galectin-3. Glycoconj J, 2008, Oct 24.

67. Suzuki O, Tasaki K, Kusakabe T, Abe M. UDPGlcNAc2-epimerase regulates cell surface sialylation and ceramide-induced cell death in human malignant lymphoma. Int J Mol Med, 22 : 339348, 2008.

68. Goni FM, Alonso A. Biophysics of sphingolipids I. Membrane properties of sphingosine, ceramides and other simple sphingolipids. Biochim Biophys Acta, 1758(12) : 1902-1921, 2006.

69. Montes LR, Ruiz-Arguello MB, Goni FM, Alonso A. Membrane restructuring via ceramide results in enhanced solute efflux. J Biol Chem, 277(14) : 11788-11794, 2002.

70. Mehlen P, Puisieux A. Metastasis : a question of life or death. Nat Rev Cancer, 6 : 449-458, 2006.

71. Swedlow SH, Campo E, Harris NL, Jaffe ES, Pileri SA, Stein H, Thiele J, Vardiman JW. WHO classification of tumors of haematopoietic and lymphoid tissues. IARC, 321-334.

72. AP1-dependent galectin-1 expression delineates classical Hodgkin and anaplastic large cell lymphomas from other lymphoid malignancies with shared molecular features. Rodig SJ, Ouyang J, Juszczynski P, Currie T, Law K, Neuberg DS, Rabinovich GA, Shipp MA, Kutok J. Clin Cancer Res, 14 : 3338-3344, 2008.

73. Juszczynski P, Ouyang J, Monti S, Rodig SJ, Takeyama K, Abramson J, Chen W, Kutok JL, Rabinovich GA, Shipp MA. The AP1-dependent secretion of galectin-1 by Reed Sternberg cells fosters immune privilege in classical Hodgkin lymphoma. Proc Natl Acad Sci USA, 104(32) : 1313413139, 2007.

74. Hoyer KK, Pang M, Gui D, Shintaku IP, Kuwabara I, Liu FT, Said JW, Baum LG, Teitell MA. An antiapoptotic role for galectin-3 in diffuse large B-cell lymphomas. Am J Pathol, 164 : 893-902, 2004. 
75. Konstantinov KN, Robbins BA, Liu FT. Galectin-3, a beta-galactoside binding animal lectin, is a marker of anaplastic large cell lymphoma. Am J Pathol, 148 : 25-30, 1996.

76. Suzuki O, Hirsch B, Abe M, Dürkop H, Stein H. Galectin-1-mediated cell death is increased by CD30-induced signaling in anaplastic large cell lymphoma cells but not in Hodgkin lymphoma cells. Lab Invest, 92(2) : 191-199, 2012.

77. ten Berge RL, Snijdewint FG, von MensdorffPouilly S, Poort-Keesom RJ, Oudejans JJ, Meijer JW, Willemze R, Hilgers J, Meijer CJ. MUC1
(EMA) is preferentially expressed by ALK positive anaplastic large cell lymphoma, in the normally glycosylated or only partly hypoglycosylated form. J Clin Pathol, 54(12) : 933-939, 2001.

78. Al-Shamkhani A. The role of CD30 in the pathogenesis of haematopoietic malignancies. Curr Opin Pharmacol, 4 : 355-359, 2004.

79. Pinter-Brown LC. SGN-30: a basis for the effective treatment of CD30 positive hematopoietic malignancies. Expert Opin Investig Drugs, 17(12) : 1883-1887, 2008. 